\title{
Estimation of performance of parts and units of a locomotive's mechanical part
}

\author{
$S$. Shantarenko ${ }^{1, *}, V$. Kuznetsov $^{1}, A$. Evseev $^{1}$, and $V$. Taranenko ${ }^{1}$ \\ ${ }^{1}$ Omsk State Transport University, 644046, Marx av., 35, Omsk, Russian Federation
}

\begin{abstract}
The paper presents the results of the development and implementation of a method for controlling technological processes of locomotive repair based on network planning, which allows monitoring and adjusting the performance of technological operations for a specific locomotive, taking into account the provision of indicators of technological preparation of repair.
\end{abstract}

\section{Introduction}

The increase in the intensity of operation, the increased requirements for the safety of train traffic and the technical condition of the rolling stock impose special requirements on the operational reliability of locomotives.

Mainline locomotives operate under conditions of negative impact of dynamic, static loads and vibration, which have a decisive influence on the nature of wear and damage to electrical and mechanical equipment. Dynamic and static loads entail significant mechanical stresses in the elements of the wheel-motor units, spring suspension, bogie and body frames, axle boxes, automatic couplers and other mechanical parts. Vibration in traction vehicles accelerates wear and increases the likelihood of malfunctions. Severe working conditions are aggravated in winter, when, due to the increase in the rigidity of the rail track, the dynamic acceleration of wheelsets from impacts at the joints reaches 15-20 g.

The specific operating conditions of traction rolling stock (TRS) impose increased requirements on its performance [1]. Ensuring the performance of locomotives is the most important task in transportation work [2].

Performance is the state of parts and assemblies, in which they are able to normally perform the specified functions with the parameters established by the regulatory and technical documentation. Performance is characterized by certain conditions (criteria), one or more of which is used in calculation [3]. The most important performance criteria are strength, rigidity, durability and reliability.

\section{Materials and methods}

Let us consider a number of criteria typical for assessing the performance of parts and units of traction rolling stock.

\footnotetext{
* Corresponding author: nich.omgups@mail.ru
} 
Strength is the ability of a part to resist fracture or plastic deformation under applied loads. Strength is the main criterion for performance, since fragile parts cannot work.

Failure of parts or assemblies leads to failure of the entire mechanical system. Strength calculations are carried out by permissible stresses $\mathrm{s} £[\mathrm{~s}], \mathrm{t} £[\mathrm{t}]$; by safety factors $\mathrm{s}^{3}[\mathrm{~s}]$; by the probability of failure-free operation $\mathrm{P}(\mathrm{t})^{3}[\mathrm{P}(\mathrm{t})]$.

In most cases, a violation of strength is considered the occurrence in the part of a stress equal to the limiting one (slim, tlim). To ensure sufficient strength (margin of safety), the following conditions must be met: $\mathrm{s} £[\mathrm{~s}]=(\operatorname{slim} /[\mathrm{s}]) ; \mathrm{t} £[\mathrm{t}]=(\mathrm{tlim} /[\mathrm{s}]) ; \mathrm{s}^{3}[\mathrm{~s}]$.

Depending on the properties of the material and the nature of the loading, the yield stress, ultimate strength (when calculating for static strength) or fatigue limit under the corresponding stress cycle (when calculating for fatigue strength - endurance) are taken as the ultimate stress [4]. When designing, it should be borne in mind that fatigue resistance is significantly reduced in the presence of stress concentrators associated with the structural form of parts (fillets, grooves, holes, etc.) or with manufacturing defects (scratches, cracks, etc.).

In some cases, parts work under loads that cause alternating contact stresses ss in the surface layers, leading to fatigue chipping of the contacting surfaces. The calculation in this case is made from the condition of endurance of the working surfaces [5].

The permissible safety factor [s] is established on the basis of the differential method as the product of partial coefficients reflecting the reliability of formulas and design loads, the homogeneity of the mechanical properties of materials, and specific safety requirements.

When calculating parts made of plastic materials under the influence of constant stresses, the permissible safety factor [s] in relation to the yield point is set to be the minimum with sufficiently accurate calculations $([\mathrm{s}]=1.3-1.5)$. The safety factor in relation to the ultimate stress $\mathrm{sU}$ when calculating parts made of brittle materials, even at constant stresses, is assigned sufficiently large $([\mathrm{s}] \geq 3)$. This is associated with the risk of destruction even if the maximum stress is once exceeded the ultimate strength. The safety factor for the endurance limit so is assigned relatively small $([\mathrm{s}]=1.5-2.5)$, since single overloads do not lead to destruction.

Stiffness is the ability of parts to resist changes in shape and size under load. The stiffness calculation provides for limiting the elastic deformations of parts within the limits permissible under specific operating conditions (for example, the quality of gearing of gear wheels and the operating conditions of bearings deteriorate with large deflections of the shafts). The importance of stiffness calculations increases due to the fact that the improvement of structural materials occurs mainly in the direction of increasing their strength characteristics ( $\mathrm{s} U$ and $\mathrm{s}-1$ ), and the elastic moduli $\mathrm{E}$ (stiffness characteristic) increase insignificantly or even remain constant. Stiffness standards are established based on operating practice and calculations. There are cases when the dimensions obtained from the strength condition are insufficient in stiffness.

Stiffness calculations are more labor intensive than strength calculations. Therefore, in a number of cases, they are limited only to the latter, but deliberately increased safety factors are taken in order to provide adequate stiffness in such an indirect way [6].

In some cases, it is necessary to take into account displacements caused not only by general, but also by contact deformations, i.e. perform calculations for contact stiffness.

Wear resistance is the property of parts to resist wear, i.e. the process of gradual change in the size and shape of parts as a result of friction. At the same time, the gaps in the kinematic pairs increase, which, in turn, leads to a violation of accuracy, the appearance of additional dynamic loads, a decrease in the cross section and, consequently, to a decrease in strength, a decrease in efficiency, and an increase in noise. At the current level of technology, about $85-90 \%$ of parts and assemblies fail as a result of wear, which causes a sharp rise in the cost of operation due to the need to periodically check their condition and 
repair. For traction rolling stock, the costs of repairs and maintenance due to wear can significantly exceed the cost of a new TRS.

Calculation of parts for wear resistance consists either in determining the conditions that ensure fluid friction (operating mode when the contacting surfaces are separated by a sufficient layer of lubricant), or in ensuring their sufficient durability by assigning appropriate permissible pressures to the sliding surfaces.

Reliability, as a criterion of operability, is estimated by the reliability coefficient $\mathrm{P}(\mathrm{t})$ the probability of maintaining operability during a given service life [7].

The probability of failure-free operation of a unit is equal to the product of the probabilities of failure-free operation of its constituent parts.

Thus, the performance of parts and assemblies of machines and mechanisms is a complex property that depends on many factors, which can be divided into three groups: design, operating conditions (modes), and the system of maintenance and repair [8].

Usually assessment of the quality of the operational functioning of structural elements of the metal structure, which include parts and assemblies of the mechanical part of the locomotive (bogies, wheel-motor units (WMU), traction motors, spring suspension, wheelsets, etc.) is carried out on the basis of the following groups of indicators:

1. Dynamic characteristics, such as: linear and angular acceleration of speed and movement of elements of metal structures; dynamic forces in ties and joints of metal structure elements; strength and the conditions for the interaction of metal structures with the external environment.

This group of indicators is determined either on the basis of the results of field experiments, or by solving mathematical models of a certain degree of complexity, describing the dynamics of a system of absolutely rigid bodies with elastic-dissipative constraints, similar to the considered metal structure.

2. Indicators of the stress state and durability of metal structures are based on local characteristics:

- distribution of stresses, shear forces and bending moments;

- a criterion for the durability of cast and welded elements of metal structures, which determines the relationship between the process of fatigue crack growth, the number of loading cycles and the level of stress state of the material in the area of the defect.

This group of indicators is calculated based on the equations of the theory of elasticity and on the basis of empirical dependencies, the parameters of which are determined from laboratory experiments.

3. The durability and reliability of the functioning of structural elements is based on the assessment of their technical condition and on the forecast of the time of failure-free operation.

The calculation is carried out on the basis of stochastic models using a priori known distribution laws of random variables. The distribution parameters reflect the operational characteristics of the metal structure.

The first two groups of indicators have a single physical essence and characterize two sides of the same process of dynamics of deformable solids. The first group of indicators represents the movement of a deformable body as an absolute solid. The second group characterizes oscillatory processes in the volume of the same body represented as a continuous medium.

As applied to metal structures, one of the main problems in the study of elastic oscillations of a continuous medium is mathematical modeling and calculation of the distribution over the volume of a solid body of a time-varying stress field.

The dynamic stress field in the elements of metal structures is formed under the influence of the forces of their interaction with the external environment and with other elements combined into a system. 
Since most structural elements can be represented in the form of rods, beams with various cross-sectional configurations, slabs and plates (or their combinations), equations for the dynamic distribution of stresses in the volume of a structural element under the action of various types of loads distributed on the surface of the element can be used as mathematical models.

The geometrical distribution of these loads (forces and moments) is determined by the location of the points of attachment of elastic links and nodes of joints that combine individual elements into a single mechanical system.

The nature of the change in surface loads over time is determined by the technical parameters of the mechanical system and its internal connections, as well as the operational parameters that determine the interaction with the external environment.

Deformation stress fields of a continuous medium are usually modeled on the basis of Newton - Euler's postulates on the change in momentum and its principal moment for the elementary volume of a continuous medium with their generalization to the entire volume occupied by the body, or using the Hamilton variational principle [9-11].

In the study of dynamic fields of elastic displacements in deformable solids, the most widespread are the Lame equations, which in expanded form are represented by the system.

$$
(\lambda+G) \frac{\partial \theta}{\partial x_{1}}+G \nabla^{2} U_{i}+X_{i} \cdot \rho=\rho \frac{\partial^{2} U_{i}}{\partial t^{2}}
$$

\section{$(\mathrm{i}=1,2,3)$}

Where $x_{i}$ - body point coordinates;

$U_{i}$ - the projection of the displacement vector of an arbitrary point on the coordinate axes;

$\theta=\operatorname{div} \bar{U}=\frac{\partial U_{1}}{\partial x_{1}}+\frac{\partial U_{2}}{\partial x_{2}}+\frac{\partial U_{3}}{\partial x_{3}}$ - relative volumetric deformation;

$\nabla^{2}=\frac{\partial^{2}}{\partial x_{1}^{2}}+\frac{\partial^{2}}{\partial x_{2}^{2}}+\frac{\partial^{2}}{\partial x_{3}^{2}}$ - second order Laplace differential operator;

$G=\frac{E}{2(1+\mu)} ; \lambda=\frac{2 \mu G}{1-2 \mu}$ - permanent reflective elastic properties of the material;

$E, \mu$ - modulus of elasticity and Poisson's ratio.

$X_{i}$ - projection of the resulting volumetric forces per unit mass;

$\rho$ - material density.

The transition to the components of the stress field in the framework of the linear Hooke theory is carried out by the formulas:

$\sigma_{i}=2 G \frac{\partial U}{\partial x_{i}}+\lambda \theta-$ for normal stress;

$\tau_{i j}=G\left(\frac{\partial U_{j}}{\partial x_{i}}+\frac{\partial U_{i}}{\partial x_{j}}\right)$ - for shear stress.

Conditions at the boundary of the continuous medium are determined by the relationship between the distribution of external forces and the distribution of stresses on the surface of the body.

For an arbitrary point $\mathrm{M}(\mathrm{x}, \mathrm{y}, \mathrm{z})$ of the surface, these dependences can be represented in matrix form.

$$
P_{n}\left(x_{1}, x_{2}, x_{3}, t\right)=T \cdot N
$$

Here $P_{n}=\left(P_{1 n}, P_{2 n}, P_{3 n}\right)^{T}$ - matrix column of projections of the normal external force on the coordinate axes; $\mathrm{T}$ - stress tensor; $\mathrm{N}=(\mathrm{n} 1, \mathrm{n} 2, \mathrm{n} 3) \mathrm{T}-$ matrix of the column of direction cosines of the normal to the surface at the point $\mathrm{M}$.

If we assume that "noticeable" changes in the initial shape of a metal structure element occur beyond the range of permissible values of elastic displacements, then the surface of 
its boundary can be unambiguously determined by its initial configuration in a coordinate system rigidly connected with a solid body.

The nature of the distribution of surface forces and the law of their change in time remain uncertain.

The place of application of surface forces and the area of their distribution are determined from the constructive arrangement of connections between the elements of the metal structure. The nature of these forces depends on the design and technical parameters of the communication devices. The law of variation of surface forces can be obtained from the solution of mathematical models of the dynamics of a metal structure as a mechanical system of absolutely rigid bodies [12].

As an example of assessing the performance of parts and assemblies of the mechanical part of a locomotive, let us perform mathematical modeling of the stress state of the suspension rod of a traction motor of a 2ES6 electric locomotive due to its cyclic loading when moving along a butt rail track.

One of the most important criteria for the performance of a structure is the strength of its parts and assemblies. Strength, as the ability of a part to resist fracture, is assessed using allowable stresses or safety margins [4].

Calculation of stresses in the position of static equilibrium was made according to the scheme shown in Figure 1.

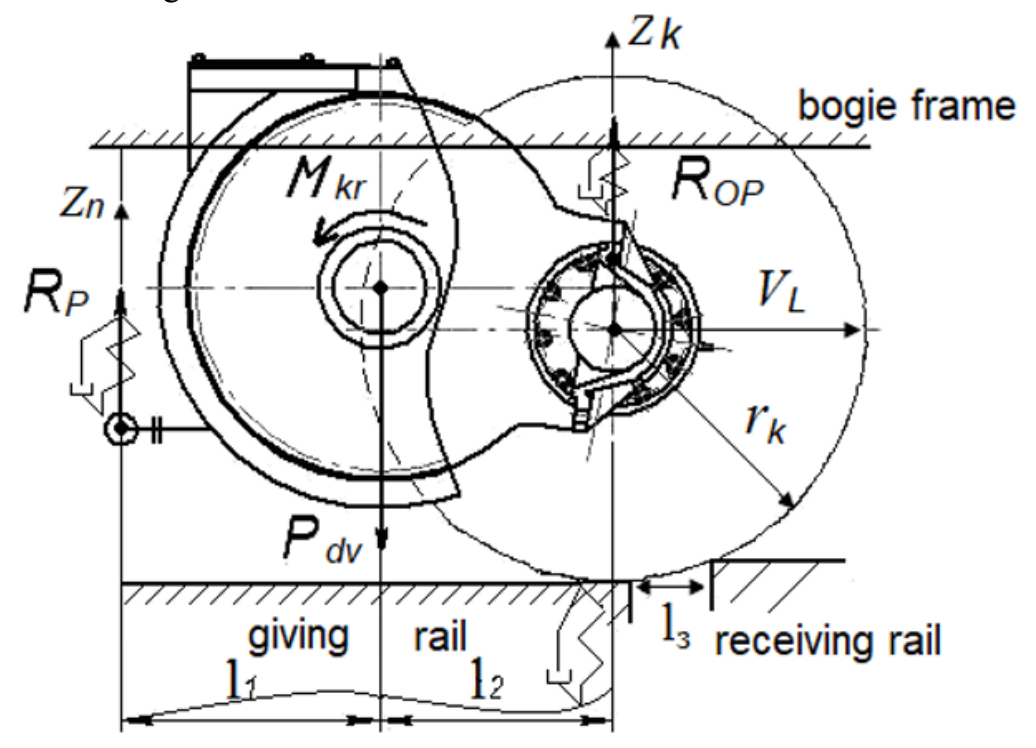

Fig. 1. Design scheme for assessing stresses in the details of the WMU: Pdv - weight of the traction motor; Rp - effort in the rod for suspending the TEM; ROP - load on the wheelset axle (reaction of the support); 11 - shoulder from the point of suspension of the traction motor to the bogie frame up to the axis of rotation of the TEM armature; 12 - shoulder from the axis of rotation of the TEM armature to the axis of the wheelset.

To assess the operational reliability of the TEM suspension elements, let's perform an analysis of the stress state of the rod material in the process of cyclic loading when moving along a butt rail track [13].

Let's compose the equilibrium equations for static forces at the WMU nodes:

$$
\left\{\begin{array}{c}
R_{P}+R_{O P}=P_{d v} \\
R_{P}\left(l_{1}+l_{2}\right)=P_{d v} l_{2},
\end{array}\right.
$$

From the system of equations (3) we obtain: 


$$
\begin{aligned}
R_{O P} & =\frac{P_{d v} l_{1}}{l_{1}+l_{2}}, \\
R_{P} & =\frac{P_{d v} l_{2}}{l_{1}+l_{2}}
\end{aligned} \quad \quad V V V
$$

For electric locomotive 2ES6 with parameters $\mathrm{Pdv}=46 \mathrm{kN} ; 11=0.642 \mathrm{~m} ; 12=0.617 \mathrm{~m}$, axle load of the wheelset $\mathrm{ROP}=23.45 \mathrm{kN}$ and the force in the traction motor suspension rod $\mathrm{RP}=22.54 \mathrm{kN}$.

The middle part of the rod is made of seamless hot-deformed pipe $89 \times 12$, steel 20 [14].

Normal stress in the cross section of the pipe [15].

$$
\sigma_{s t}=\frac{R_{P}}{S}
$$

where

$$
S=\frac{\pi\left(d_{e x}^{2}-d_{i n}^{2}\right)}{4}
$$

- area of the road pipe cross-section;

$\mathrm{d}_{\mathrm{e} x}=89 \mathrm{~mm}$ - external and $\mathrm{d}_{\mathrm{i} n}=12 \mathrm{~mm}$ - internal pipe diameters, respectively;

$\mathrm{S}=6105 \mathrm{~mm} 2$.

According to formula (6), the normal stress in the rod material

$\sigma_{\text {st }}=3.69 \frac{\mathrm{N}}{\mathrm{mm}^{2}}$.

For seamless hot-deformed pipe made of steel 20, endurance limit $\sigma_{V}=193 \frac{\mathrm{N}}{\mathrm{mm}^{2}}$, which exceeds the stress from static load by more than 50 times [16].

However, this fact does not give grounds for a conclusion about the operational reliability of the rod, since the decisive factor for the survivability of the material of the metal structure is the cyclic change in stress. Therefore, it is necessary to study the dynamics of stress changes in the rod material when an electric locomotive moves along a rail track.

We obtain a mathematical model of the dynamic behavior of a traction motor under operating conditions according to the design scheme shown in Figure 1.

Since the purpose of mathematical modeling is to assess the maximum stresses arising in the details of the WMU of the electric locomotive 2ES6 under the influence of joints and irregularities of the rail track, therefore, the design scheme (Figure 1) did not take into account the oscillations of the bogie frame jumping and galloping, due to their lesser effect on the arising stresses (the vibration frequency of the bogie frame is much less than the vibration frequency of the WMU during the movement of the electric locomotive).

We choose the following as generalized coordinates:

$\mathrm{Zk}$ - vertical displacements of the center of gravity of the wheelset relative to the position of static balance;

$\mathrm{Zn}$ - movement of the bracket for attaching the rod to the TEM relative to the center of the bracket in the unloaded state.

We compose expressions for the kinetic $\mathrm{T}$, potential $\mathrm{P}$ energies and the scattering function F:

$$
\begin{gathered}
T=\frac{1}{2} m_{d} \dot{Z}_{d}^{2}+\frac{1}{2} m_{k} \dot{Z}_{k}^{2}+\frac{1}{2} J_{d} \dot{\phi}_{d}^{2} \\
P=\int_{0}^{z_{K}} F_{R} d z_{k}+\int_{0}^{\Delta_{\Pi}} F_{P} d \Delta_{P}+\int_{0}^{z_{H}} F_{T} d z_{n} \\
F=\int_{0}^{\dot{z}_{K}} \beta_{R} \dot{z}_{k} d \dot{z}_{k}+\int_{0}^{\dot{z}_{K}} \beta_{P} \dot{z}_{k} d \dot{z}_{k}+\int_{0}^{\dot{z}_{H}} \beta_{T} \dot{z}_{n} d \dot{z}_{n} .
\end{gathered}
$$


The dissipative forces of energy dissipation in the rail track, spring suspension and in the elastic element of the traction motor suspension rod are taken linear with respect to the speeds of the corresponding displacements.

Potential forces can also be represented by linear functions:

$$
F_{R}=Z H_{R} z_{k}
$$

- spring suspension elastic force;

$$
F_{T}=Z H_{T} z_{n}-P_{T}
$$

- elastic force in the rod of the TEM suspension;

$$
F_{P}=Z H_{P}\left(z_{k}-\eta\right)
$$

- rail deflection elastic force;

$$
\eta=H \sin \frac{2 \pi}{L_{H}} x
$$

- undulating unevenness of the track along the length of the rail,

where $\mathrm{H}, \mathrm{LH}-$ amplitude and length of rail unevenness;

$\mathrm{x}$ - rail length;

ZHR, $\beta \mathrm{R} ; \mathrm{ZHT}, \beta \mathrm{T}$; ZHP, $\beta \mathrm{P}$ - elastic and dissipative coefficients in the spring suspension, in the elastic element of the traction motor suspension and in the rail track, respectively;

$\mathrm{md}, \mathrm{mk}$ - mass of traction motor and wheelset;

$\mathrm{Jd}$ - moment of inertia of traction motor.

Static load on the TEM suspension rod

$$
P_{T}=\frac{P d v l_{2}}{l_{1}+l_{2}}
$$

For a traction motor, the displacement of the center of gravity zd and rotation relative to the central transverse axis $\varphi d$ are expressed through the generalized coordinates:

$$
\begin{gathered}
z_{d}=\frac{z_{n} l_{2}+z_{k} l_{1}}{l_{1}+l_{2}} ; \\
\phi_{d}=\frac{z_{k}-z_{n}}{l_{1}+l_{2}} .
\end{gathered}
$$

Using the Lagrange equations of the second kind, a mathematical model of the dynamic behavior of the WMU elements was obtained:

$$
\frac{\partial}{\partial t}\left(\frac{\partial T}{\partial \dot{q}}\right)+\frac{\partial P}{\partial q}+\frac{\partial F}{\partial \dot{q}}=0
$$

Thus:

$$
\begin{gathered}
m_{k} \ddot{z}_{k}-\frac{m_{d} l_{1}}{\left(l_{1}+l_{2}\right)^{2}}\left(\ddot{z}_{n} l_{2}+\ddot{z}_{k} l_{1}\right)+\frac{J_{d}}{\left(l_{1}+l_{2}\right)^{2}}\left(\ddot{z}_{k}-\ddot{z}_{n}\right)+ \\
+Z H_{R} z_{k}+Z H_{P}\left(z_{k}-\eta\right)+\dot{z}_{k}\left(\beta_{R}+\beta_{P}\right)=0 \\
\frac{m_{d} l_{2}}{\left(l_{1}+l_{2}\right)^{2}}\left(\ddot{z}_{n} l_{2}+\ddot{z}_{k} l_{1}\right)-\frac{J_{m}}{\left(l_{1}+l_{2}\right)^{2}}\left(\ddot{z}_{k}-\ddot{z}_{n}\right)+\beta_{T} \dot{z}_{n}+Z H_{T} z_{n}-P_{d} \frac{l_{2}}{l_{1}+l_{2}}=0 .
\end{gathered}
$$


Bringing similar ones and solving the resulting system of equations with respect to $\ddot{z}_{k}$ and $\ddot{z}_{n}$, we get:

$$
\begin{gathered}
\ddot{z}_{k}\left(a_{11} a_{22}-a_{12}^{2}\right)+z_{k} Z H_{k} a_{22}-z_{n} Z H_{T} a_{12}+\dot{z}_{k} \beta_{k} a_{22}-\dot{z}_{n} \beta_{T} a_{12}= \\
=a_{22} Z H_{P} \eta+a_{12} \frac{P_{d} l_{2}}{l_{1}+l_{2}} \\
\ddot{z}_{n}\left(a_{11} a_{22}-a_{12}^{2}\right)+z_{n} Z H_{T} a_{11}-z_{k} Z H_{k} a_{12}+\dot{z}_{n} \beta_{T} a_{11}-\dot{z}_{k} \beta_{k} a_{12}= \\
=-a_{11} P_{d} \frac{l_{2}}{l_{1}+l_{2}}-a_{12} Z H_{n} \eta
\end{gathered}
$$

where $Z H_{k}=Z H_{R}+Z H_{n}$;

$\beta_{k}=\beta_{R}+\beta_{n}$

$a_{11}=\frac{m_{d} l_{1}^{2}+J_{d}+m_{k}\left(l_{1}+l_{2}\right)^{2}}{\left(l_{1}+l_{2}\right)^{2}}$;

$a_{22}=\frac{m_{d} l_{2}^{2}+J_{d}}{\left(l_{1}+l_{2}\right)^{2}}$

$a_{12}=\frac{m_{d} l_{1} l_{2}-J_{d}}{\left(l_{1}+l_{2}\right)^{2}}$.

To obtain a solution, we determine the initial conditions and external influences.

At $\mathrm{t}=0$, we can take:

$z_{k}=\dot{z}_{k}=0 ; z_{n}=\frac{P_{d} l_{2}}{\left(l_{1}+l_{2}\right) Z H_{T}} ; \dot{z}_{n}=0$.

An external effect in the case of a butt rail track will be the increment in the speed of the center of gravity of a wheelset when passing butt irregularities [17].

With an accuracy sufficient for engineering calculations, the vertical component of the speed of the center of gravity of the wheelset can be taken [18]

$$
V_{V}=\frac{V_{L}}{r_{K}} l_{Z}
$$

where VL- locomotive speed,

$\mathrm{rK}$ - wheel rolling radius,

$1 Z$ - butt gap.

Oscillation of the wheelset $\mathrm{z}$, caused by the action of the butt unevenness, is completely damped after three to four periods due to dissipative forces.

Therefore, it is possible to calculate the dynamic equations along the length of one irregularity with the initial conditions at $\mathrm{t}=0$ :

$z_{n}=\dot{z}_{n}=0 ; z_{k}=0 ; \dot{z}_{k}=\frac{V_{L} l_{z}}{r_{K}}$.

The impacts of butt irregularities are repeated after a period of time $\tau=\frac{L_{R}}{V_{L}}$, where LR rail length.

The dynamic component of the longitudinal force FT, acting in the rod of the TEM suspension

$$
F_{T}=Z H_{T} \cdot Z_{n}
$$

To assess the durability of the rod material of the traction motor suspension, it is necessary to select the highest FTmax and the smallest FTmin values of this force over time $\tau$.

Then the normal stresses in the cross section of the suspension rod

$$
\sigma{\frac{F_{T \max }}{S} \max }
$$




$$
\sigma \frac{F_{T \min }}{S} \min
$$

where $\mathrm{S}$ - area of the rod cross-section.

In this case, the characteristics of the loading cycle are the following values:

$$
\sigma_{m}=\frac{\sigma_{\max }+\sigma_{\min }}{2}
$$

- medium stress;

$$
\sigma_{N}=\frac{\sigma_{\max }-\sigma_{\min }}{2}
$$

- stress amplitude;

$$
\sigma_{A}=\frac{\sigma_{\min }}{\sigma_{\max }}
$$

- asymmetry coefficient.

The durability of the suspension rod is assessed by the fatigue curve of the metal from which it is made.

For steel 20, the fatigue curve is shown in Figure 2 [19].

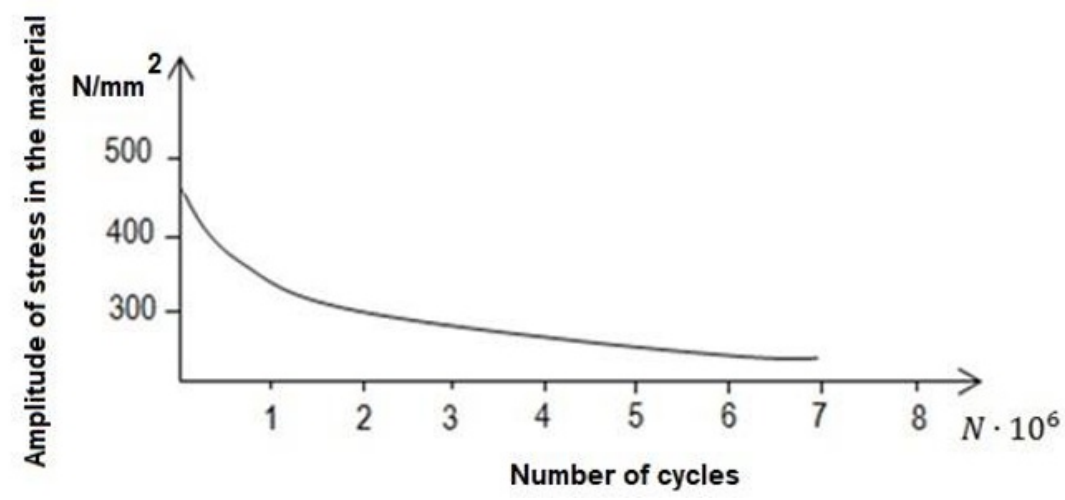

Fig. 2. Fatigue curve for steel 20.

If we take for VL the average speed of the electric locomotive over the time $\mathrm{T}$, then the number of loading cycles of the traction motor suspension rod [20]

$$
n=\frac{T \cdot V_{L}}{L_{R}}
$$

where $\mathrm{L}_{\mathrm{R}}$ - rail length.

The performance of the rod is assessed by the value of the stress amplitude in the suspension rod and the number of loading cycles along the fatigue curve (Figure 2).

Theoretical studies carried out as a result of the numerical integration of the obtained mathematical model showed that when an electric locomotive moves along a butt rail track, significant longitudinal forces FT arise in the suspension rod of a traction electric motor.

$\sigma_{\text {max }}$ In this case, the stresses in the material of the traction motor suspension rod reach values close to critical (Figure 3 ). 


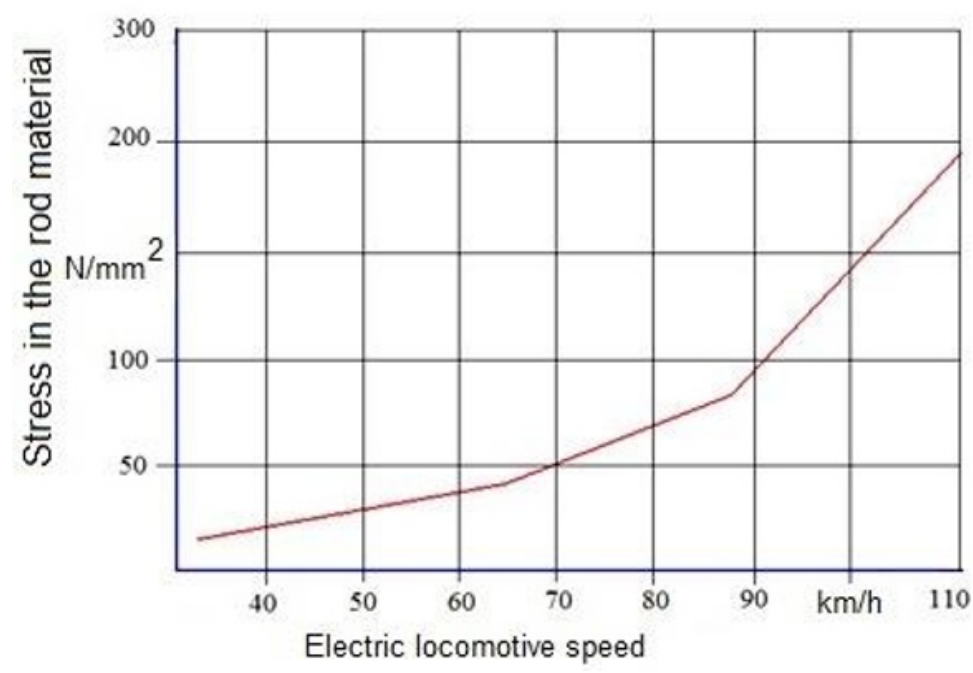

Fig. 3. Dependence of stresses $\sigma_{\max }$ in the TEM suspension rod on the speed of the electric locomotive

The maximum stress $\sigma_{\max }$ at an electric locomotive speed of $110 \mathrm{~km} / \mathrm{h}$ is 190.45 $\mathrm{N} / \mathrm{mm} 2$. This value is close to the endurance limit $(193 \mathrm{~N} / \mathrm{mm} 2)$ for the material from which the rod is made (steel 20, seamless hot-deformed pipe).

\section{Results}

Mathematical modelling was carried out without taking into account possible stress concentrators in the material, therefore, the probability of destruction of the leash in operating conditions is high.

Thus, the proposed mathematical models and calculation formulas make it possible to assess the stresses arising in the parts and assemblies of the mechanical part of the locomotive in operation, which makes it possible to determine the operability of the structure and develop proposals for reducing the negative impact of dynamic loads on the mechanical part and the drive.

\section{References}

1. V.A. Chetvergov, Reliability of locomotives: a textbook for higher educational institutions of the railway transport (M.: Marshrut, 2003)

2. S.G. Shantarenko, Principles for assessing the quality of traction rolling stock functioning in the structure of the transportation process on railway, Proceedings of the VIII International Scientific and Practical Conference "Science and Education 2005", Dnipropetrovsk National University of Railway Transport 60, 57 - 60 (2005)

3. A.N. Savoskin, Strength and reliability of the rolling stock of railways (M.: Mashinostroenie, 1990)

4. P.A. Stepin, Resistance of materials: a textbook for mechanical engineering specialties of universities (7th ed. M.: Higher school, 1983)

5. M.N. Ivanov, Machine parts: textbook for students of higher technical educational institutions (5th ed., Rev. M.: Higher school, 1991) 
6. G.B. Iosilevich, Applied mechanics: textbook for universities (M.:Higher school, 1989)

7. GOST 27.002-89. Reliability in technology. Terms and Definitions (M.: Publishing house of standards, 1980)

8. V.G. Grigorenko, Improving the efficiency of locomotive use under operating conditions on the railways of the Eastern region of Russia: thesis in the form of scientific report of Doctor in Engineering: 05.22.07 (Omsk, 1999)

9. J.S. Slattery, The theory of transfer of momentum, energy and mass in continuous media (Moscow: Energiya 1978)

10. N.I. Bezukhov, Fundamentals of the theory of elasticity, plasticity and creep (M: Higher school. 1961)

11. N.P. Abovskiy, N.P. Andreev, A.P. Deruga, Variational principles of the theory of elasticity and theory of shells (Moscow: Nauka, 1978)

12. N.A. Kilchevsky, Course of theoretical mechanics 2, 544(1977)

13. S.G. Shantarenko, Mathematical modeling of the stress state of the suspension of the traction motor of the electric locomotive, Journal of the Rostov State University of Railways 2 (58), 46 - 51 (2015)

14. GOST 8732-78. Seamless hot-deformed steel pipes. Range (M.: Standartinform, 2007)

15. N.I. Bezukhov, N.I. Fundamentals of the theory of elasticity, plasticity and creep (M.: Higher school, 1961)

16. V.G. Sorokin, Grade guide of steels and alloys (M.: Mashinostroenie, 1989)

17. V.I. Kiselev, The regularity of the occurrence of vibro-shock oscillations in traction electric drives of locomotives, Thesis of Dr. in Engineering (Moscow, 1992)

18. M.P. Pakhomov, Assessment of the level of impulse influence of rail joints on a locomotive wheel, Interaction of rolling stock and tracks, dynamics of locomotives: Interuniversity collection of scientific works, Omsk State Transport University 128, 9-16 (1971)

19. N.M. Belyaev, Resistance of materials (M.: Publishing house "Nauka", 1965)

20. A.P. Pavlenko, Dynamics of traction drives of main locomotives (M.: Mashinostroenie, 1991)

21. I.I. Galiev, On the longitudinal dynamics of bogies, Railway transport 5, 38-40 (2009)

22. V Yu Alpatov and M I Balzannikov 2020 IOP Conf. Ser.: Mater. Sci. Eng. 913 032057. doi:10.1088/1757-899X/913/3/032057

23. V Yu Alpatov and S M Petrov 2020 IOP Conf. Ser.: Mater. Sci. Eng. 913042037. doi:10.1088/1757-899X/913/4/042037 